\title{
Evaluation of the Effects of Paclobutrazol and Cultivation Years on Saponins in Ophiopogon japonicus Using UPLC-ELSD
}

\author{
Peng Sun, Juhua Tong, and Xianen Li iD \\ Institute of Medicinal Plant Development, Chinese Academy of Medical Sciences and Peking Union Medical College, \\ Beijing 100193, China \\ Correspondence should be addressed to Xianen Li; xianenli@yeah.net
}

Received 18 March 2020; Revised 31 May 2020; Accepted 18 June 2020; Published 6 July 2020

Academic Editor: Eladia M. Pena Mendez

Copyright (c) 2020 Peng Sun et al. This is an open access article distributed under the Creative Commons Attribution License, which permits unrestricted use, distribution, and reproduction in any medium, provided the original work is properly cited.

Nowadays, there is a growing concern about the quality of herbs used in traditional Chinese medicine. In this study, we evaluated the impacts of paclobutrazol and cultivation period on steroid saponins in Ophiopogon japonicus. A rapid method to simultaneously determine three principle steroid saponins (ophiopogonins $\mathrm{B}, \mathrm{D}$, and $\mathrm{D}^{\prime}$ ) using ultraperformance liquid chromatography combined with an evaporative light-scattering detector was developed. The contents of three saponins in paclobutrazoltreated and nontreated Sichuan O. japonicus and those in the 2-year and 3-year Zhejiang O. japonicus were analyzed. The results showed that the saponin contents were sharply reduced in paclobutrazol-treated O. japonicus as compared to the control, whereas the concentrations of the three targeted saponins in Zhejiang O. japonicus varied with the increase in cultivation years, reflecting varied effects on saponins. Our study provided chemical evidences for further quality control and agricultural practices of O. japonicus.

\section{Introduction}

In recent decades, there has been a growing popularity of herbal medicines, which can provide polypharmacological effects that orthodox medicines cannot deliver and have been indicated to have distinct therapeutic effects on the treatment of some chronic diseases and metabolic syndromes [1]. It was estimated that $80 \%$ of the world's population use herbal medicines as their source of primary care [2]. With the increasing interest in traditional Chinese herbal medicines, the concerns regarding their quality, efficacy, and safety also arise $[3,4]$. One critical issue facing traditional Chinese medicines is the different concentrations and proportions of constituents in herbal products, which may lead to great variations in therapeutic results and safety issues. To minimize variation in final herbal products, standardization of procedures should cover the entire field of study from cultivation of herbs to clinical application [5]. And so, the factors that make up the variability in herbs, such as cultivation practice and genotype, must be comprehensively evaluated in the cultivation process.
Ophiopogon japonicus (L.f.) Ker Gawl is an evergreen perennial herb in the family Liliaceae that is widely distributed in South China. O. japonicus is commonly known as "maidong" in Chinese, and its tuberous roots have long been used to treat coughs, sore throats, thirstiness, constipation, and insomnia in traditional Chinese medicine $[6,7] . O$. japonicus is largely produced in Zhejiang and Sichuan Province in China, where O. japonicus is usually called Zhe maidong (ZMD) and Chuan maidong (CMD), respectively. Though ZMD and CMD both originated from O. japonicus, they display different appearances and qualities. Both ZMD and CMD have a yellowish spindle-like shape, but the former is usually thinner and darker than the latter. To distinguish between ZMD and CMD and evaluate their qualities, several analytic and pharmacological studies had been carried out [8-12]. These studies exhibited that CMD and ZMD possessed different chemical constituents and pharmacological activities that would play pivotal roles in clinical efficacy of $O$. japonicus and its derived preparations, implying that the geographical origin of O. japonicus is a key factor for the differences in CMD and ZMD. 
Besides geographical location, other factors including cultivation practice and genotype also significantly affect the quality of herbs, as has been observed in several herbal species $[13,14]$. This is also the case in O. japonicus. In practice, ZMD demands a long growing period to be harvested, which is usually harvested in the third year after planting, while CMD is often harvested in the second year as which grows faster in Sichuan. Furthermore, CMD is usually treated with plant regulator paclobutrazol during its growth cycle to achieve high yielding and good commercial grade. Paclobutrazol is a kind of plant regulator that can reduce plant height, improve stem diameter and leaf number, alter root architecture, and directly contribute to yield increase [15]. Currently, the effects of agronomic factors on $O$. japonicus have attracted researchers' interests. Li et al. and Lin et al.'s studies showed that fertilizers and regulators influenced the agronomic character, yield, and appearance quality of $O$. japonicus [16, 17]. Among the agronomic factors, paclobutrazol plays a prominent role in regulation of the growth and metabolism of O. japonicus. Li et al. and Zhan et al. reported that paclobutrazol not only affected the growth characteristics of aerial parts but also significantly improved the tuberous root number, size, and dry matter $[18,19]$. Furthermore, two recent studies have demonstrated that application of paclobutrazol influenced the total contents of saponins, flavonoids, and polysaccharides (i.e., the main active compositions in O. japonicus) [20, 21]. However, the study on the impact of agricultural practices on $O$. japonicus is still limited. It will be interesting and important to differentiate the detailed differences of constituents caused by the influencing factors.

Steroid saponins are the mostly studied active ingredients in O. japonicus, and this group of compounds exhibits broad biological activities such as cardiovascular protection, anti-inflammation, anticancer, antioxidation, immunomodulation, and antitussive [22]. According to the Chinese Pharmacopoeia (2015 Edition), the total content of saponins (calculated as ruscogenin) is used as a quality control index of O. japonicus and the UV spectrometric method is stipulated for the quantitative determination of the total content of saponins [23]. As many substances in plants have UV-like absorption contours, UV spectrophotometry cannot serve as an ideal approach for the identification of steroid saponins, and there has been increased interest in establishing more precise and accurate methods for the quantification of saponins in O. japonicus in recent years. Presently, highperformance liquid chromatography (HPLC) remains the most common technique for the quality assessment of $O$. japonicus. Several HPLC-based methods to determine steroid saponins have been reported $[8,24,25]$. However, the separation of steroid saponins using HPLC (concerning resolution and analysis time) was not ideal due to their highly similar structures and high molecular weights, which cannot well meet the fast and accurate requirements for the quality assessment of $O$. japonicus from different sources. Hence, it is crucial to establish a rapid and reliable analytical method to analyze the steroid saponins in O. japonicus. Ultraperformance liquid chromatography (UPLC) has now become a powerful analytical technique that enables to rapidly separate targeted compounds with higher sensitivity and selectivity than HPLC. More recently, UPLC tandem with time-of-fight mass spectrometry has been used to identify isomers of saponins from O. japonicus [26]. In the present study, a rapid ultraperformance liquid chromatography with evaporative light-scattering detection (UPLCELSD) method for simultaneous determination of ophiopogonins $\mathrm{B}, \mathrm{D}$, and $\mathrm{D}^{\prime}$ was established; this method was validated and then successfully used for evaluation of the effects of paclobutrazol and cultivation years on saponin contents. The results would broaden our knowledge on the phytochemical variation in cultivated O. japonicus under different cultivation practices.

\section{Materials and Methods}

2.1. Plant Materials. CMD and ZMD were, respectively, collected from Santai county, Sichuan Province, and Cixi city, Zhejiang Province of China. CMDs were divided into two groups: paclobutrazol-treated group and control group. In the first year, the paclobutrazol group was sprayed with paclobutrazol in October. Two groups of CMDs were harvested in the second year. ZMDs were divided into two types: the yellow-leafed type (YL) and green-leafed type (GL). YL O. japonicus is a variant of GL O. japonicus (the most common O. japonicus). ZMDs were separately harvested in the second and third years. All samples were washed, deenzymed at $105^{\circ} \mathrm{C}$ for $15 \mathrm{~min}$, and then dried at $60^{\circ} \mathrm{C}$. All plant materials were authenticated by Prof. Xianen Li (Institute of Medicinal Plant Development, Chinese Academy of Medical Sciences). The information about all the samples mentioned above is summarized in Table 1.

2.2. Chemicals and Regents. The reference compounds ophiopogonin B and ophiopogonin $\mathrm{D}^{\prime}$ were purchased form Sichuan Weikeqi Biotech Co., Ltd. (China), while ophiopogonin D was from Shanghai Yilin Biotech Co., Ltd. (China). The purity of standards was higher than 98\%. HPLC grade acetonitrile was from Fisher Scientific (USA). Water used for all analyses was from Wahaha Co. (China). The methanol and butanol used for plant extraction were of AR grade from Beijing Chemical Corporation (Beijing, China). All other chemicals were of reagent grade.

2.3. Standard Preparation. The reference compounds ophiopogonins $\mathrm{B}, \mathrm{D}$, and $\mathrm{D}^{\prime}$ were accurately weighed and dissolved in $1 \mathrm{~mL}$ methanol to produce a mixed standard stock solution with the concentrations of $0.2013 \mathrm{mg} / \mathrm{mL}$, $0.2813 \mathrm{mg} / \mathrm{mL}$, and $0.2125 \mathrm{mg} / \mathrm{mL}$, respectively. $2,4,8,12$, 16 , and $20 \mu \mathrm{L}$ of the standard mixture were separately taken and diluted in methanol for UPLC-ELSD analysis to make calibration curves.

2.4. Sample Preparation. Two grams of O. japonicus powder was extracted under reflux with $100 \mathrm{~mL}$ methanol for 1 hour and filtered. The extract was evaporated to dryness in a rotary evaporator at $65^{\circ} \mathrm{C}$ under reduced pressure and 
TABLE 1: Summary of the samples used in the present study.

\begin{tabular}{lcccc}
\hline Origin & Genotype & Developmental stage & Paclobutrazol treatment & No. of samples \\
\hline \multirow{2}{*}{ CMD } & \multirow{2}{*}{ GL } & 2nd year & Yes & No \\
& & & No & 10 \\
\hline \multirow{3}{*}{ ZMD } & \multirow{2}{*}{ GL } & 2nd year & No & 5 \\
& & 3rd year & No & 10 \\
3 & No \\
\hline
\end{tabular}

redissolved in $50 \mathrm{~mL}$ of water. The obtained solution was subsequently extracted four times with water-saturated butanol $(25 \mathrm{~mL}, 25 \mathrm{~mL}, 20 \mathrm{~mL}$, and $20 \mathrm{~mL}$ each). The pooled butanol portions were washed twice with $5 \mathrm{~mL} 10 \%$ ammonium hydroxide solution. The butanol fraction was then evaporated to dryness at $65^{\circ} \mathrm{C}$ under the reduced pressure and dissolved in $5 \mathrm{~mL}$ methanol for the below UPLC-ELSD analysis.

2.5. UPLC Conditions. A Waters Acquity UPLC system (Waters, USA, including Waters 2707 autosampler and Empower 2 chromatography workstation) coupled with an evaporative light-scattering detector was used for sample analysis. The separation of samples was performed on an Acquity UPLC BEH C18 column $(2.1 \mathrm{~mm} \times 100 \mathrm{~mm}, 1.7 \mu \mathrm{m}$, Waters, USA) maintained at $30^{\circ} \mathrm{C}$. The injection volume was $5 \mu \mathrm{L}$. The mobile phase consisted of (A) acetonitrile and (B) water. The UPLC elution conditions were optimized as follows: $45 \%$ A $0-1 \mathrm{~min}, 45 \%-49 \%$ A $1-3 \mathrm{~min}, 49 \%$ A $3-5 \mathrm{~min}$, $49 \%-51 \%$ A $5-8 \mathrm{~min}, 51 \%$ A $8-9 \mathrm{~min}$, and $51 \%-55 \%$ A $9-$ $10 \mathrm{~min}$, and then, the column was returned to $45 \% \mathrm{~A}$ for the next injection. The flow rate was set at $0.20 \mathrm{~mL} / \mathrm{min}$. The ELSD settings were as follows: drift tube temperature, $90^{\circ} \mathrm{C}$; nitrogen flow rate, $2.07 \mathrm{~mL} / \mathrm{min}$; and heating power level of the sprayer, $60 \%$. Three replicates of each sample were measured.

\section{Results and Discussion}

3.1. Method Development. Like almost all other saponins, ophiopogonins lack a chromophore. Although saponins absorb UV light at a wavelength below $210 \mathrm{~nm}$, due to their low concentrations, they were undetectable in O. japonicus samples under the condition of UV detection at $208 \mathrm{~nm}$ (Figure 1). In literature, quantification analysis of saponins in O. japonicus was generally performed by means of HPLCELSD detection based mainly on C18 columns under gradient elution [8, 24, 25]. However, because of the high similarity of their chemical structures, performing a baseline separation of the ophiopogonins using the HPLC method was found to be difficult. Even under conditions of long elution time and high flow rate, the peaks were not well resolved, making the HPLC method time-consuming and imprecise. In the present study, the developed UPLC method ensured sufficient chromatographic separation and accurate and precise quantification of ophiopogonins $\mathrm{B}, \mathrm{D}$, and $\mathrm{D}^{\prime}$ (Figure 2). Moreover, the use of ELSD detector, a common detector in most labs, makes the developed method suitable for routine quality control of $O$. japonicus.
At the stage of the method development, the extraction methods: heat reflux extraction, maceration, and ultrasoundassisted extraction, were applied for the extraction of saponins from O. japonicus. A maximum saponin yield of $0.274 \mathrm{mg} / 2 \mathrm{~g}$ DW could be obtained in $2 \mathrm{~h}$ by heat reflux extraction $(2 \mathrm{~g}$ of sample: $100 \mathrm{~mL}$ of methanol, $70^{\circ} \mathrm{C}$ ) compared to $24 \mathrm{~h}$ for maceration $(0.185 \mathrm{mg} / 2 \mathrm{~g}$ DW; $2 \mathrm{~g}$ of sample: $100 \mathrm{~mL}$ of methanol, $25^{\circ} \mathrm{C}$ ) and $2 \mathrm{~h}$ ultrasound-assisted extraction $(0.257 \mathrm{mg} / 100 \mathrm{mg} \mathrm{DW} ; 2 \mathrm{~g}$ of sample: $100 \mathrm{~mL}$ of methanol, $25^{\circ} \mathrm{C}$ ). Therefore, the heat reflux extraction technique, which achieved the best yields of saponins without consuming too much time, is suitable compared with maceration and ultrasound-assisted extraction methods. In heat reflux extraction using water, ethanol, and methanol solvents, methanol displayed better extraction efficiency $(0.131 \mathrm{mg} / \mathrm{g})$ than ethanol and water, the yield obtained from water extraction was especially low $(0.056 \mathrm{mg} / \mathrm{g})$, and thus, methanol was selected as extraction solvent. Moreover, there was no correlation between the extraction time ( $1 \mathrm{~h}, 2 \mathrm{~h}, 3 \mathrm{~h}$, and $4 \mathrm{~h})$ and the extraction yield of saponins; $1 \mathrm{~h}$ was found to be adequate for the analysis. And so, heat reflux extraction using $100 \mathrm{ml}$ methanol for $1 \mathrm{~h}$ was adopted in this study for the subsequent analysis.

3.2. Methods Validation. The current UPLC-ELSD method was validated for its linearity, precision, repeatability, and stability effects. The linear regression equations obtained for three analytes were $y=1.6489 x+5.1185$ (ophiopogonin $\mathrm{B}$, $R^{2}=0.9994$ ),$\quad y=1.4937 x+5.3108 \quad$ (ophiopogonin $\mathrm{D}$, $R^{2}=0.9992$ ), and $y=1.5438 x+5.2992$ (ophiopogonin $\mathrm{D}^{\prime}$, $\left.R^{2}=0.9993\right)$, respectively, suggesting a good relationship between concentrations and peak areas of the analytes within the test ranges. A sample was assayed successively for 6 times to evaluate the instrumental precision. The RSDs of peak areas were less than $3 \%(2.61 \%, 2.42 \%$, and $1.80 \%$, respectively), indicating that the analysis instrument was in good precision condition. Six samples from the same batch were prepared and analyzed by the developed method for repeatability assessment; the RSDs for ophiopogonin B, ophiopogonin D, and ophiopogonin $\mathrm{D}^{\prime}$ were $1.76 \%, 2.04 \%$, and $2.57 \%$, respectively, indicating that the developed method was with acceptable repeatability. For the stability test, the RSDs of peak areas for ophiopogonins B, D, and $\mathrm{D}^{\prime}$ detected by the method were $2.36 \%, 2.93 \%$, and $2.05 \%$ within $24 \mathrm{~h}$, respectively, reflecting that this method was stable. The established method also had acceptable accuracy with spike recovery, which were $99.63 \%, 101.67 \%$, and $100.45 \%$ for ophiopogonins B, D, and $\mathrm{D}^{\prime}$, respectively. Finally, this method was successfully employed for 


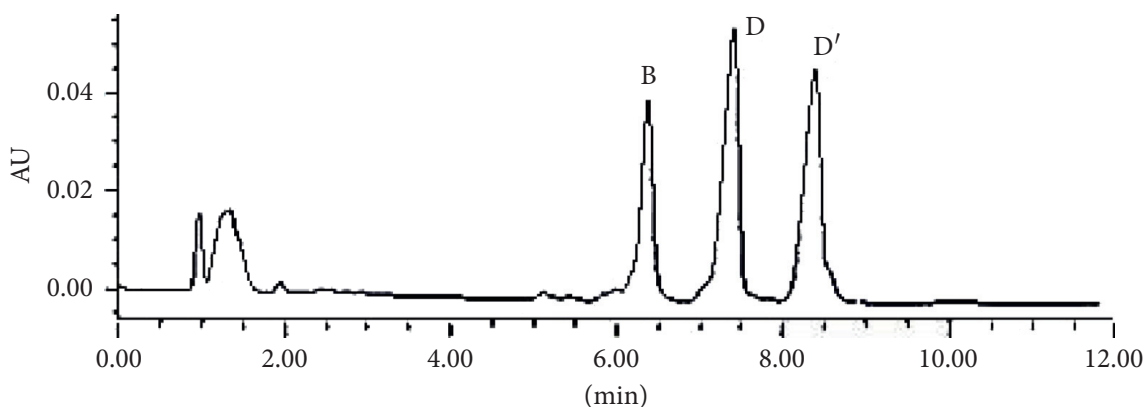

(a)

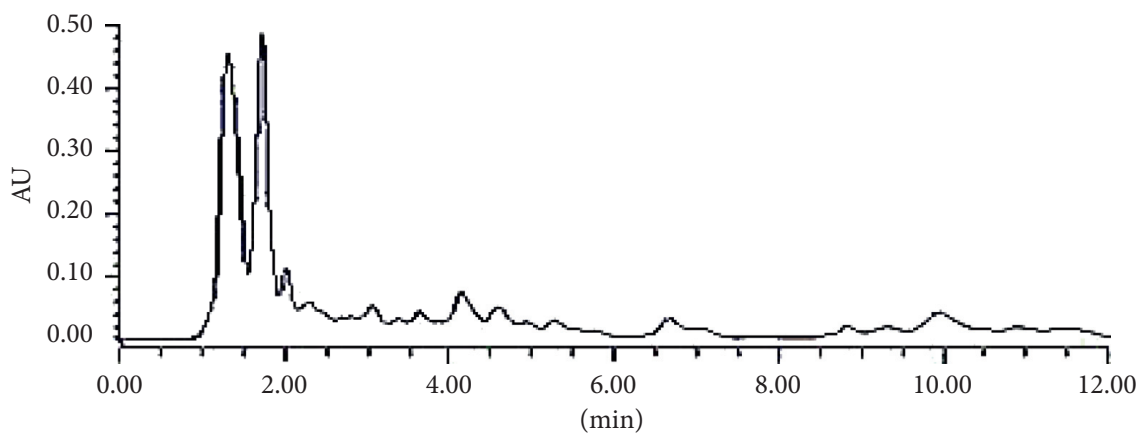

(b)

FIgURE 1: UPLC-UV chromatographs of standard mixture (a) and maidong extract (b) at $208 \mathrm{~nm}$.

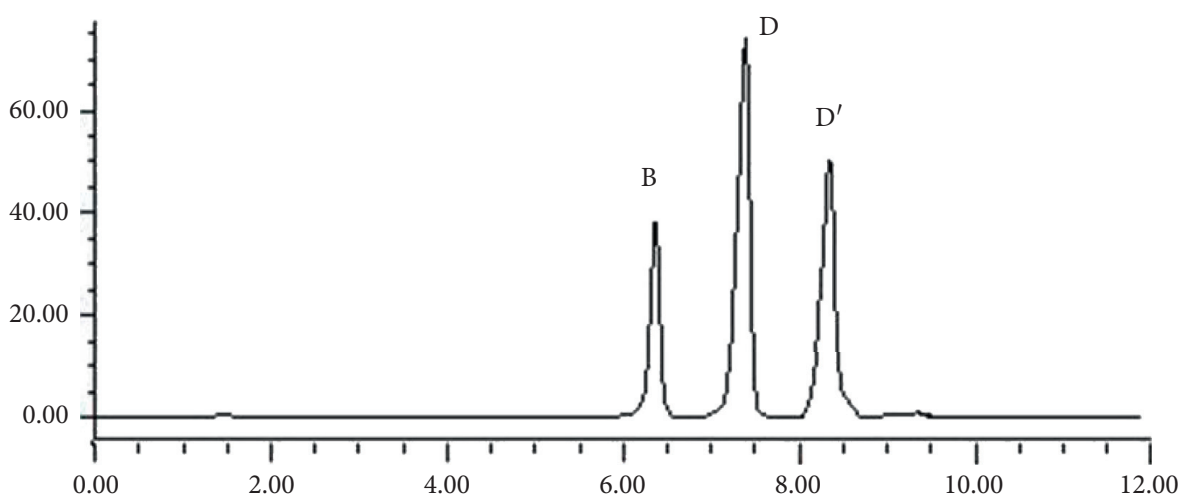

(a)

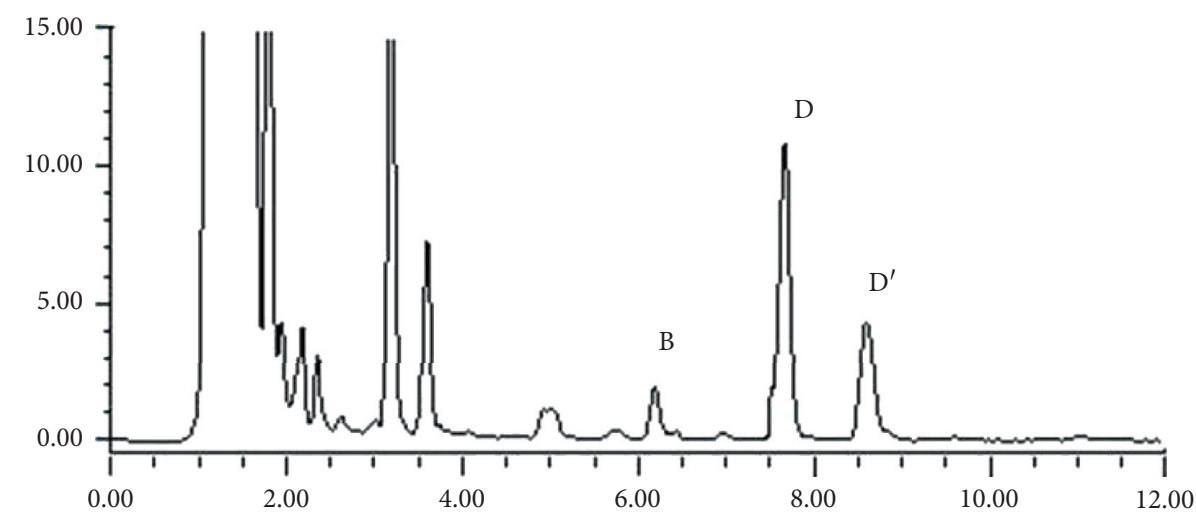

(b)

Figure 2: Continued. 


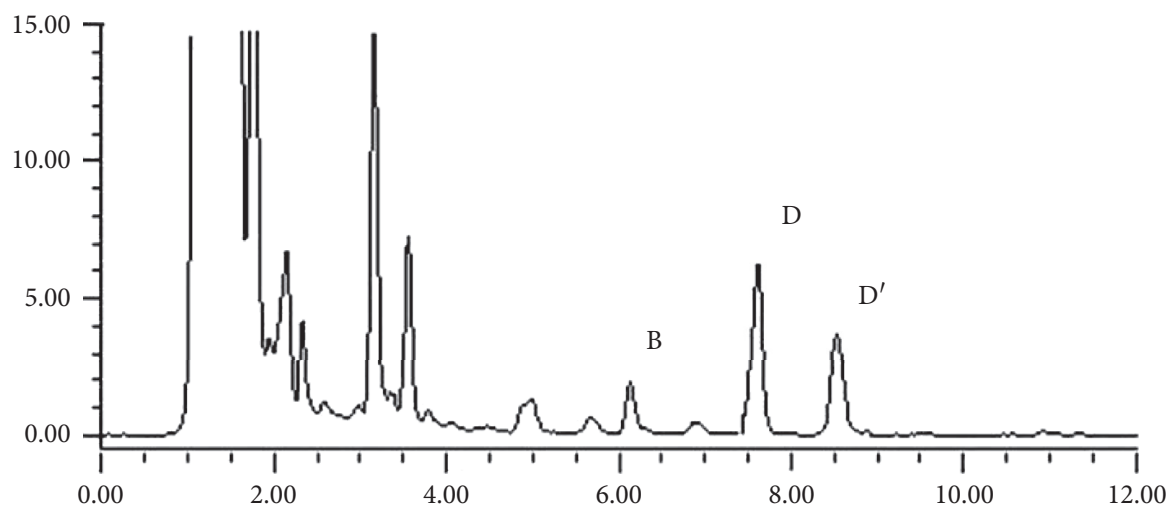

(c)

Figure 2: UPLC-ELSD chromatographs of standard mixture (a), CMD extract (b), and ZMD extract (c).

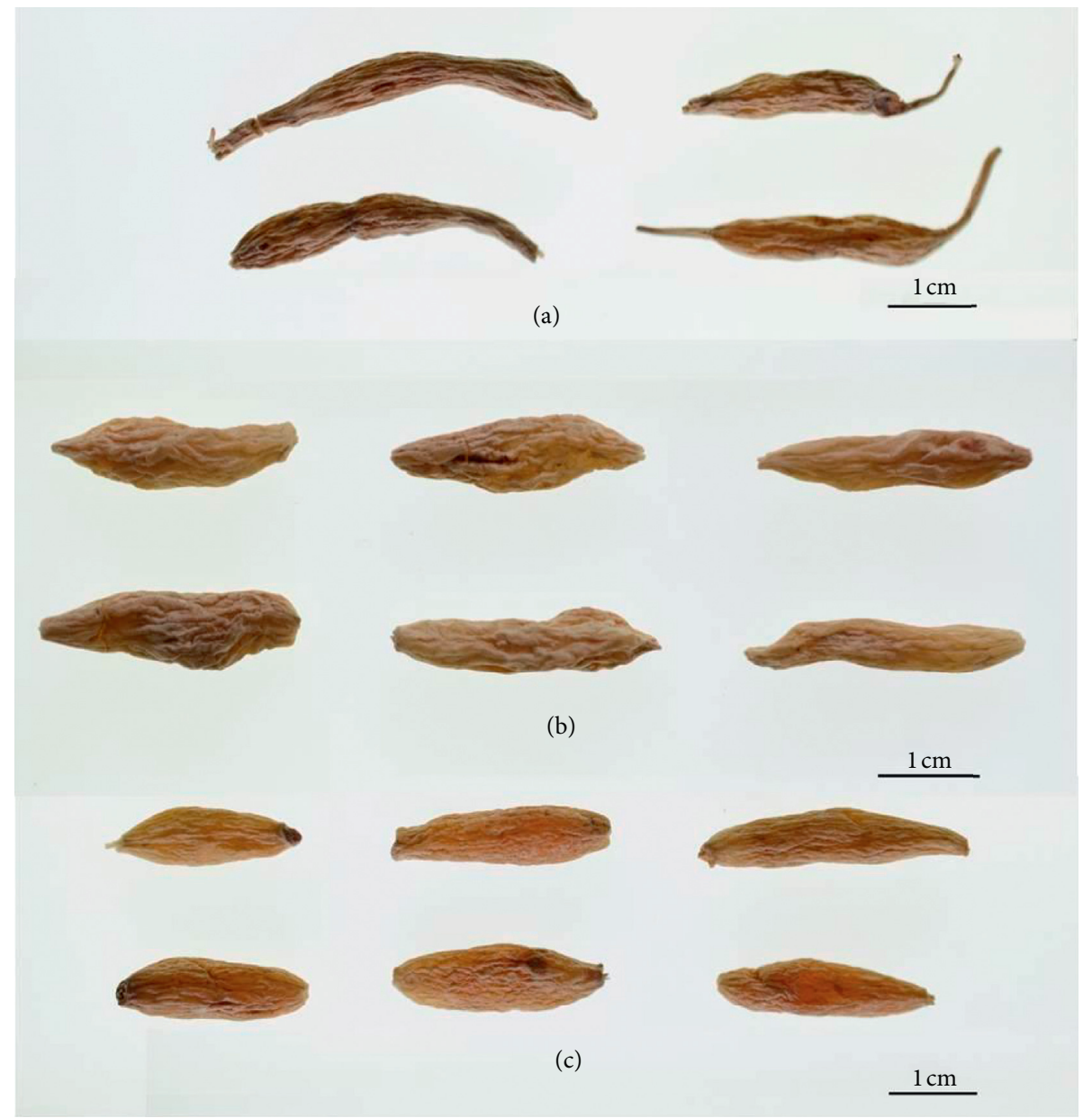

FIgURE 3: Samples of ZMD (a), CMD (b), and paclobutrazol-treated CMD (c).

quantitative determination of ophiopogonins $\mathrm{B}, \mathrm{D}$, and $\mathrm{D}^{\prime}$ in $\mathrm{CMD}$ and $\mathrm{ZMD}$ samples.

3.3. Effect of Paclobutrazol on Saponin Contents. In the decade, CMD is becoming the dominant species in maidong market due to its high yield and appearance quality. During growing season, paclobutrazol is often applied on CMD to achieve economic efficiency. Paclobutrazol-treated CMD samples have a glossy appearance and are plumper compared with nonpaclobutrazol-treated CMD samples, as shown in Figure 3. However, the influence of paclobutrazol on the effective constituents in O. japonicus needs to be 


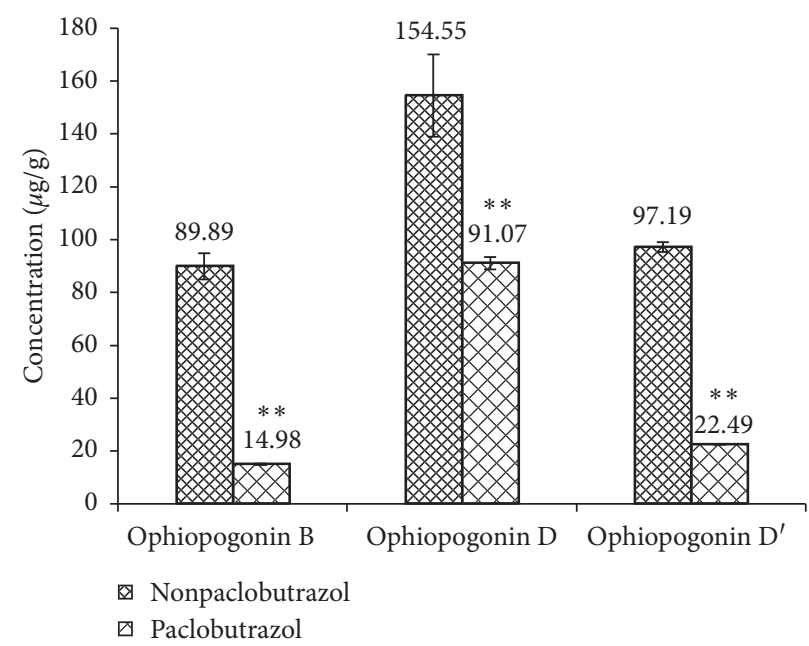

FIGURE 4: The concentrations of ophiopogonin B, ophiopogonin D, and ophiopogonin $\mathrm{D}^{\prime}$ in paclobutrazol-treated CMD and nontreated samples. ${ }^{* *} p \leq 0.01$.

TABLE 2: The mean contents of saponins in the 2-year and 3-year ZMD samples ( $\mu \mathrm{g} / \mathrm{g})$.

\begin{tabular}{|c|c|c|c|c|}
\hline & \multicolumn{2}{|c|}{ GL } & \multicolumn{2}{|c|}{ YL } \\
\hline & $2 \mathrm{Y}$ & $3 \mathrm{Y}$ & $2 \mathrm{Y}$ & $3 \mathrm{Y}$ \\
\hline Ophiopogonin B & $52.64 \pm 5.45$ & $36.79 \pm 0.22$ & $16.33 \pm 0.84$ & $17.56 \pm 0.32$ \\
\hline Ophiopogonin D & $18.64 \pm 0.60$ & $41.51 \pm 2.12^{* *}$ & $2.45 \pm 0.72$ & $11.29 \pm 0.38^{* *}$ \\
\hline Ophiopogonin $\mathrm{D}^{\prime}$ & $62.02 \pm 10.34$ & $65.90 \pm 2.64$ & $14.32 \pm 0.29$ & $15.63 \pm 0.35$ \\
\hline Total & 133.33 & 144.20 & 33.10 & 44.48 \\
\hline
\end{tabular}

** Significant difference in contents between 2-year and 3-year maidong ( $p \leq 0.01$ ). GL: green leaf maidong; YL: yellow leaf maidong; 2 Y: 2-year maidong; 3Y: 3 -year maidong.

comprehensively evaluated for medicinal purpose. In this study, we assessed the effect of paclobutrazol on the major saponins in O. japonicus using UPLC-ELSD. The concentrations of ophiopogonins $\mathrm{B}, \mathrm{D}$, and $\mathrm{D}^{\prime}$ in blank and paclobutrazol-treated CMD samples were determined and shown in Figure 4. The results showed that the contents of three saponins in CMD were dramatically reduced by using paclobutrazol, especially for those of ophiopogonins $\mathrm{B}$ and $\mathrm{D}^{\prime}$, which, respectively, decreased by $83.33 \%$ and $76.86 \%$ as compared to the controls. These findings suggest that paclobutrazol plays a significantly negative role in contributing to the accumulation of three targeted saponins in O. japonicus. It is also of no surprise that paclobutrazol-treated CMDs had much lower total saponin content than controls, and the average concentrations of treatment group and the control group were $128.54 \mu \mathrm{g} / \mathrm{g}$ and $341.63 \mu \mathrm{g} / \mathrm{g}$, respectively. This is consistent with the previous studies reported by Lin et al. and Zhang et al. in which the total saponin contents in $O$. japonicus were reduced by the application of paclobutrazol $[20,21]$.

3.4. Influence of Growing Period on the Saponin Contents of $Z M D s$. The varied amounts of three saponins in the 2-year and 3-year ZMDs are shown in Table 2. The contents of ophiopogonin D in O. japonicus sharply increased with the cultivation years, in which GL O. japonicus increased from $18.64 \mu \mathrm{g} / \mathrm{g}$ in the 2 nd year to $41.51 \mu \mathrm{g} / \mathrm{g}$ in the $3 \mathrm{rd}$ year and YL O. japonicus was from $2.45 \mu \mathrm{g} / \mathrm{g}$ in the 2 nd year to $11.29 \mu \mathrm{g} / \mathrm{g}$ in the $3 \mathrm{rd}$ year. It seemed that the increase in growth period had a very weak effect on the accumulation of ophiopogonin $\mathrm{D}^{\prime}$, which slightly increased in both types of O. japonicus within the 3 rd year. As for ophiopogonin B, the content variation associated with growth was significantly different in the two genotypes. The mean amount of ophiopogonin B in GL $O$. japonicus declined from $52.64 \mu \mathrm{g} / \mathrm{g}$ in the 2 nd year to $36.79 \mu \mathrm{g} / \mathrm{g}$ in the $3 \mathrm{rd}$ year, while that in YL O. japonicus slightly increased from $16.33 \mu \mathrm{g} / \mathrm{g}$ in the 2nd year to $17.56 \mu \mathrm{g} / \mathrm{g}$ in the $3 \mathrm{rd}$ year. Besides, it is noteworthy that ophiopogonins $\mathrm{B}, \mathrm{D}$, and $\mathrm{D}^{\prime}$ were lower in $\mathrm{YL} O$. japonicus compared with GL O. japonicus, suggesting the impact of genotype on saponins.

Our results revealed that cultivation period and genotype played a definite role in influencing the content of saponins in O. japonicus, providing important information on the standardization of cultivating O. japonicus. However, the interaction effects of cultivation period, genotype, paclobutrazol, and other environmental factors such as geographical location on the accumulation of saponins in ZMD and CMD are still not clear, and this will be one of the important research aspects that needs more detail investigation in future. 


\section{Conclusions}

A reliable UPLC-ELSD method was established to simultaneously determine ophiopogonins $\mathrm{B}, \mathrm{D}$, and $\mathrm{D}^{\prime}$ in Ophiopogon japonicus. Our results demonstrated that the paclobutrazol treatment sharply decreased the content of ophiopogonins $\mathrm{B}, \mathrm{D}$, and $\mathrm{D}^{\prime}$ in CMDs. The content of ophiopogonin $\mathrm{D}$ in the 3-year ZMDs was significantly higher than that in the 2-year ZMDs for both genotypes. As for ophiopogonins $\mathrm{B}$ and $\mathrm{D}^{\prime}$, the two genotype $\mathrm{ZMDs}$ showed variable changes in response to growth. Our results also provided the information on the quality control of $O$. japonicus by using standard cultivation practices and selecting desirable varieties containing a higher amount of desired saponins in future.

\section{Data Availability}

The data used to support the findings of this study are available from the corresponding author upon request.

\section{Conflicts of Interest}

The authors declare that there are no conflicts of interest in publication of this paper.

\section{Acknowledgments}

This work was supported by the National Science and Technology Major Project (2012ZX09304006).

\section{References}

[1] S. Edwards, I. Da-Costa-Rocha, M. J. Lawrence, C. Cable, and M. Heinrich, "Use and efficacy of herbal medicines: part 2-clinical effectiveness," Pharmaceutical Journal, vol. 289, p. 270, 2012.

[2] M. Ekor, "The growing use of herbal medicines: issues relating to adverse reactions and challenges in monitoring safety," Frontiers in Pharmacology, vol. 4, p. 177, 2014.

[3] WHO, WHO Traditional Medicine Strategy 2002-2005, WHO, Geneva, Switzerland, 2002.

[4] P. K. Mukherjee and P. J. Houghton, Evaluation of Herbal Medicinal Products: Perspectives on Quality, Safety and Efficacy, Pharmaceutical Press, London, UK, 2009.

[5] D. Ghosh, "Quality issues of herbal medicines: internal and external factors," International Journal of Complementary \& Alternative Medicine, vol. 11, no. 2, Article ID 00350, 2018.

[6] X. Y. Sun, F. Yu, W. Xiao, L. J. Xu, and P. G. Xiao, "Research progress on modern application of Ophiopogonis Radix," Modern Chinese Medicine, vol. 20, no. 11, pp. 1453-1458, 2018.

[7] X. Lin, Q. F. Zhou, and D. S. Xu, "Research progress of the pharmacological actions of Ophiopogon root," Shanghai Journal of Traditional Chinese Medicine, vol. 38, no. 6, pp. 59-61, 2004.

[8] X.-E. Li, Y.-X. Wang, P. Sun, and D.-Q. Liao, "Determination of saponin content in hang maidong and chuan maidong via HPLC-ELSD analysis," Journal of Analytical Methods in Chemistry, vol. 2016, Article ID 7214607, 5 pages, 2016.
[9] M. Tan, J. Chen, C. Wang et al., "Quality evaluation of Ophiopogonis Radix from two different producing areas," Molecules, vol. 24, no. 18, pp. 1-13, 2019.

[10] Y. Ge, X. Chen, D. Gođevac et al., "Metabolic profiling of saponin-rich ophiopogon japonicus roots based on $1 \mathrm{H}$ NMR and HPTLC platforms," Planta Medica, vol. 85, no. 11/12, pp. 917-924, 2019.

[11] Y. Lin, D. Zhu, J. Qi, M. Qin, and B. Yu, "Characterization of homoisoflavonoids in different cultivation regions of Ophiopogon japonicus and related antioxidant Activity," Journal of Pharmaceutical and Biomedical Analysis, vol. 52, no. 5, pp. 57-762, 2010.

[12] M. Zhao, W.-f. Xu, H.-y. Shen et al., "Comparison of bioactive components and pharmacological activities of ophiopogon japonicas extracts from different geographical origins," Journal of Pharmaceutical and Biomedical Analysis, vol. 138, pp. 134-141, 2017.

[13] A. Szakiel, C. Pączkowski, and M. Henry, "Influence of environmental abiotic factors on the content of saponins in plants," Phytochemistry Reviews, vol. 10, no. 4, pp. 471-491, 2011.

[14] C. V. Borges, I. O. Minatel, H. A. Gomez-Gomez, and G. P. .P. Lima, "Medicinal plants: influence of environmental factors on the content of secondary metabolites," in Medicinal Plants and Environmental Challenges, M. Ghorbanpour and A. Varma, Eds., Springer, Cham, Switzerland, 2017.

[15] W. Tesfahun, "A review on: response of crops to paclobutrazol application," Cogent Food \& Agriculture, vol. 4, no. 1, pp. 1-9, 2018.

[16] S. J. Li, Z. M. Yang, F. Y. Lei et al., "Effects of combined application of organic and inorganic fertilizers on agronomic traits, yield and quality of Ophiopogon japonicus," Soils and Fertilizers Sciences in China, no. 4, pp. 67-77, 2019.

[17] Q. X. Lin, M. Li, H. Y. Luo, and X. Huang, "Study on the influence of CPPU on Chuanmaidong growth and development," Pharmacy and Clinics of Chinese Materia Medica, vol. 5, no. 1, pp. 6-8, 2014.

[18] Q. X. Lin, M. Li, Y. H. Luo, X. Huang, and B. Y. Yang, “A preliminary study on the effect of plant growth regulator on growth and development of Ophiopogon japonicus," Lishizhen Medicine and Materia Medica Research, vol. 25, no. 8, pp. 1994-1996, 2014.

[19] N. Zhan, S. Y. Tao, and M. C. Xie, "Effects of MET on growth, development and yield of Dwarf Lilyturf Ophiopogon japonicus (L.f.) Ker-Gawl," Hubei Agricultural Sciences, vol. 53, no. 12, pp. 2835-2838, 2014.

[20] Q. X. Lin, M. Li, H. Y. Zhou, C. Liu, M. Ren, and C. Y. Zhao, "'Study of the influence of plant growth regulators on Ophiopogon japonicus's total saponins and total polysaccharide content," Modern Chinese Medicine, vol. 16, no. 5, pp. 399-402, 2014.

[21] Y. Q. Zhang, S. J. Li, Q. L. Deng, Q. S. Wen, Y. P. Fu, and X. F. Chen, "Effects of combined application of chemical fertilizers and plant growth retardants on yield and formation of secondary metabolites of Ophiopogon japonicus," Pratacultural Science, vol. 36, no. 6, pp. 1544-1552, 2019.

[22] M.-H. Chen, X.-J. Chen, M. Wang, L.-G. Lin, and Y.-T. Wang, "Ophiopogon japonicus-A phytochemical, ethnomedicinal and pharmacological review," Journal of Ethnopharmacology, vol. 181, pp. 193-213, 2016.

[23] Chinese Pharmacopoeia Commission, Chinese Pharmacopoeia, Vol. 1, Chemical Industry Press, Beijing, China, 2015.

[24] Q. Yong, K. Zhang, B. Li, Y. A. Tang, and X. Mei, "Study on the determination method of Ophiopogonin $\mathrm{D}$ in 
Ophiopogonis Radix," Journal of Bethune Medical Science, vol. 15, no. 4, pp. 417-419, 2017.

[25] F. M. Wu, X. Y. Cai, P. Wang, X. H. Bao, M. Li, and J. Zhou, "HPLC simultaneous determination of contents of 5 saponin constituents in Ophiopogonis Radix," China Journal of Chinese Materia. Medica, vol. 40, pp. 4022-4025, 2015.

[26] R. Y. Yan, F. X. Ma, H. S. Yu et al., "Identification of steroidal saponins in Ophiopogonis Radix by UPLC-Q-TOF-MSE combined with relative retention time," Chinese Journal of Experimental Traditional Medical Formulae, vol. 22, no. 24, pp. 43-50, 2016. 